\title{
Herramientas para la enseñanza de la visualización y diseño molecular de metabolitos y macromoléculas
}

\author{
Tools for teaching molecular design and visualization of \\ metabolites and macromolecules
}

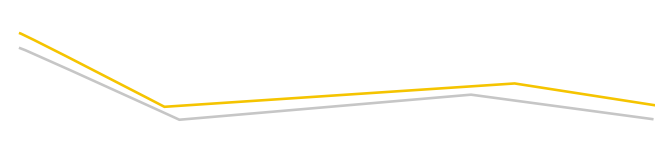

Walter Josué Hernández-Santos*, **, Blanca Estela Barrera-Figueroa*, Francisco Noé Mendoza**, Julián Mario Peña-Castro*®

\begin{abstract}
Hernández-Santos, W. J., Barrera-Figueroa, B. E., Mendoza, F. N., \& Peña-Castro, J. M. (2017). Herramientas para la enseñanza de la visualización y diseño molecular de metabolitos y macromoléculas. Investigación y Ciencia de la Universidad Autónoma de Aguascalientes, $25(71)$, 45-51.
\end{abstract}

RESUMEN

La visualización digital de moléculas y macromoléculas de los sistemas biológicos se utiliza de forma profesional en publicaciones e investigaciones científicas para describir sus propiedades químicas y físicas en tres dimensiones. En el aula, estas herramientas pueden facilitar al estudiante la comprensión de conceptos complejos de la estructura molecular en química y biología. En este trabajo se reporta un curso de visualización molecular digital, su manual (UNPA, s. f.) y una estrategia de implementación dirigida a $-\mathrm{y}$ probada con- docentes de nivel medio-superior. Con el uso de paquetes de cómputo y bases de datos profesionales de libre acceso o mediante licencias académicas, se logró ilustrar diversos conceptos de química y biología que se enseñan en los programas educativos de los profesores asistentes. Adicionalmente, mediante una estrategia de investigación, los docentes lograron planear, desarrollar y exponer imágenes autogeneradas de di-

Palabras clave: PyMOL; Marvin; proteínas; enzimas; ácidos nucleicos.

Keywords: PyMOL; Marvin; protein; enzyme; nucleic acids.

Recibido: 15 de noviembre de 2016, aceptado: 5 de julio de 2017

* Laboratorio de Biotecnología Vegetal, Instituto de Biotecnología, Universidad del Papaloapan. Av. Circuito Central 200, Colonia Parque Industrial, C. P. 68301, San Juan Bautista Tuxtepec, Oaxaca, México. Correo electrónico: wsantos@ibt.unam.mx; bbarrera@unpa.edu.mx; julianpc@unpa.edu.mx

** Laboratorio de Química Teórica, Instituto de Química Aplicada, Universidad del Papaloapan. Av. Circuito Central 200, Colonia Parque Industrial, C. P. 68301, San Juan Bautista Tuxtepec, Oaxaca, México. Correo electrónico: fmendoza@unpa.edu.mx

$凶$

Autor para correspondencia ferentes biomoléculas de su interés y útiles para su práctica docente.

ABSTRACT

The digital visualization of molecules and macromolecules from biological systems is professionally employed in scientific research and publications to depict their chemical and physical properties in three dimensions. In the classroom, these tools can help the student to improve his understanding of complex concepts in structural chemistry and biology. In this work, it is reported a molecular visualization course, an accompanying manual (UNPA, s. f.), and an implementation strategy aimed for and tested with preuniversity teachers. Using professional software and databases of open access or academically licensed, we assembled demonstrations illustrating a diversity of chemistry and biology concepts commonly reviewed in courses taught by the assistants. Additionally, through a research strategy, the assistants achieved planning, development and exposition of self-generated images of their interest and useful in their teaching practice.

INTRODUCCIÓN

Las proteínas y los ácidos nucleicos son macromoléculas, a través de ellas fluye la información para construir a los seres vivos (Peña-Castro, GregorioRamírez, \& Barrera-Figueroa, 2013). En la concepción tridimensional de las macromoléculas está la clave de muchas de sus propiedades químicas y biológicas. Por ejemplo, en el arreglo tridimensional de los residuos de aminoácidos de las proteínas, se puede encontrar el fundamento de su papel como catali- 
IIVESTIGACIÓn Y CIERCIA DE LA UNIVERSIDAD AUTÓNOMA

46

\section{DE AGUASCALIERTES}

zadores bioquímicos o como hormonas. En el caso de los ácidos nucleicos, en su tridimensionalidad está codificada su capacidad de almacenar y transmitir información genética.

Dichas moléculas se estudian en todos los programas de educación preparatoria. De tal forma, existe la necesidad de hacer accesibles las herramientas que ayuden en el aula a transitar de las ilustraciones de macromoléculas como objetos en dos dimensiones (figura 1) a 3D, a crear material práctico para comprender la información sobre sus propiedades tridimensionales y a conocer su tamaño en relación con sustratos u otras moléculas.

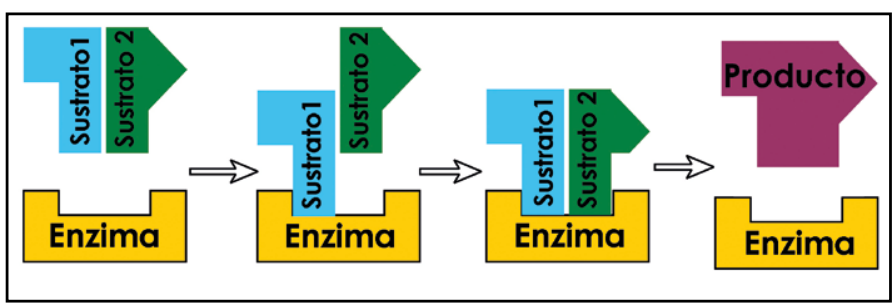

Figura 1. Modelo en dos dimensiones que se utiliza comúnmente en libros de texto para ilustrar una reacción enzimática. Elaboración propia.

Desde hace décadas, los especialistas en biología molecular y bioquímica han desarrollado diferentes modelos físicos para visualizar estructuras e interacciones moleculares nano y femtométricas con fines educativos (Vázquez-Contreras, 2015). Con el advenimiento de herramientas digitales, este objetivo se ha vuelto más sencillo y su empleo en el aula cuenta con una opinión positiva de los maestros y alumnos (Badotti et al., 2014; Vázquez-Contreras, 2015; White, Kahriman, Luberice, \& Idleh, 2010). No obstante lo anterior, los manuales de uso de estas herramientas informáticas —en inglés o español- describen las capacidades de los paquetes a una audiencia familiarizada en bioinformática y biología compleja de las moléculas (DeLano, \& Bromberg, 2004; Gonçalves de Almeida, 2012; Reyes-Trejo, 2013; Sánchez-Murcia, \& Gago-Badenas, 2016), no para alguien que busca una primera aproximación a esta herramienta de enseñanza y que requiere instrucciones detalladas para desarrollar sus materiales, por ejemplo, profesores e instructores preuniversitarios.

En este trabajo se presentan un manual y una estrategia para apoyar al maestro de nivel educativo preparatoria en la enseñanza de las estructuras de las moléculas de la vida en 3D. Mediante el uso de herra- mientas disponibles en internet, el docente aprenderá a generar sus propias estructuras moleculares de interés y modelarlas en $3 \mathrm{D}$. También será capaz de utilizar modelos de macromoléculas reportados en bases de datos de uso internacional, para destacar en ellas las zonas donde reside su función biológica. Se preparó un manual que incluye instrucciones desde la descarga e instalación de los paquetes informáticos, uso y funciones principales, hasta la explicación bioquímica de las estructuras de las moléculas e indicaciones para lograr visualizaciones que destaquen su principal función en 3D.

Los paquetes bioinformáticos que se usaron son Marvin y PyMOL. El primero permite dibujar y modelar moléculas pequeñas. El segundo, es una herramienta de visualización de estructuras micro y macromoleculares (metabolitos, ácidos nucleicos y proteínas) determinadas experimentalmente por difracción de rayos $X$ o resonancia magnética nuclear. También se explica el uso del servidor I-TASSER que predice estructuras tridimensionales, así como bases de datos que permiten hacer investigaciones originales sobre estructuras químicas.

\section{MAIERIALES Y MÉEIODOS}

\section{Software}

Para la edición digital de estructuras químicas y su transformación en archivos de coordenadas 3D se usó el paquete MarvinSketch que pertenece a Marvin Suite (Marvin, 16.3.21). Es un software comercial de la compañía ChemAxon y se puede obtener una versión licenciada sin costo para uso académico por 2 años renovable en su portal (ChemAxon Ltd., s. f.). Para la visualización de la estructura de las moléculas pequeñas y macromoléculas se utilizó PyMOL, un paquete comercial de la compañía Schrödinger LLC, que también cuenta con una licencia sin costo de uso educativo descargable desde su portal (Schrödinger LLC, s. f.). Los detalles de instalación, solicitud de licencias educativas, estructura de la interfaz de usuario, guías paso a paso, y resultados de ejercicios, se encuentran en el manual Visualización de biomoléculas con herramientas computacionales, con ISBN 978-607-96428-5-3, descargable en la página de la Universidad del Papaloapan (Hernández-Santos, W. J., Mendoza, N., Barrera-Figueroa, B. E., \& Peña-Castro, J. M., 2016).

\section{Bases de datos}

A través del portal de internet Worldwide Protein Data Bank se empleó la base de datos Protein Data Bank (Berman et al., 2000; wwPDB, s. f.) para buscar estruc- 


\section{IIVESTIGAGIÓn Y CUERCIA DE LA UחIVERSIDAD AUTÓNOMA DE AGUASCALIERTES}

turas tridimensionales de proteínas y de enzimas de interés educativo con base en la guía de actualización solicitada por el Instituto de Estudios de Bachillerato del estado de Oaxaca (IEBO). Desde el PDB se descargaron archivos de coordenadas en formato PDB y la fuente bibliográfica original para desarrollar la enseñanza a través de actividades de investigación dirigida. También se emplearon bases de datos de literatura científica públicas como NCBI (s. f.) y privadas como Scopus (s. f.) y Web of Science (2017) para encontrar reportes técnicos y números de accesión PDB que documenten estructuras involucradas en algún fenómeno de interés del profesor.

\section{Modelado en línea}

Para el modelado de proteínas en 3D a partir de una secuencia primaria se utilizó el servidor I-TASSER (Yang et al., 2014; Zhang Lab, 2017), portal académico gratuito bajo registro de usuario que utiliza un algoritmo que supone que pequeñas secuencias de proteínas comparten una estructura tridimensional, busca patrones de semejanza en la proteína problema, los compara con los depositados en PDB (2-7 días) y brinda un modelo que se puede visualizar con PyMOL. Para ilustrar su funcionamiento se utilizó la secuencia de un alfa-amilasa de Bacillus amyloliquefasciens (NCBI: JTJG00000000) por ser una enzima con amplias aplicaciones industriales y muchos homólogos reportados en el PDB.

\section{Estrategia de trabajo}

El manual se probó en una estrategia de trabajo con un grupo de 26 maestros del IEBO, provenientes de todas las regiones de Oaxaca, en forma de curso de actualización en biología de 30 h (figura 2). La colección de diapositivas que se usó en las sesiones teóricas que se mencionan en la figura 2 se encuentra disponible para descarga en la misma dirección electrónica del manual.

Las actividades se realizaron en un salón de cómputo con computadoras de 2 GB de RAM y procesador Intel Core 2 Duo en entorno Windows XP, con acceso a internet y el auxilio de un proyector digital. El curso comenzó con la instalación de los paquetes de cómputo y la explicación de la importancia y límites de las licencias académicas. Posteriormente, con las moléculas pequeñas se revisaron de forma teórica los conceptos preseleccionados sobre la base del programa de estudios del IEBO (tabla 1). Continuó el uso de Marvin Sketch y la generación de moléculas tridimensionales y ejercicios que se detallan en el manual. El uso de I-TASSER se ilustró por el instructor debido a que sólo se puede hacer una tarea por dirección IP y toma de 2 a 7 d. Se alternaron sesiones teóricas y prácticas para revisar macromoléculas (tabla 1 y figura 2) que se seleccionaron a partir del programa de estudios del IEBO.

\begin{tabular}{|c|c|c|c|c|c|}
\hline & Lunes & Martes & Miércoles & Jueves & Viernes \\
\hline $8: 00-9: 00$ & $\begin{array}{c}\text { Inauguración de } \\
\text { curso. }\end{array}$ & $\begin{array}{l}\text { III. Estructura de } \\
\text { biomoléculas, } \\
\text { Cristalografía de } \\
\text { Rayos X y PDB.org }\end{array}$ & $\begin{array}{c}\text { Visualización digital } \\
\text { de } \\
\text { macromoléculas: } \\
\text { Insulina y } \\
\text { Anticuerpo contra } \\
\text { cocaína. }\end{array}$ & $\begin{array}{l}\text { IX. Biotecnología } \\
\text { molecular. }\end{array}$ & $\begin{array}{l}\text { Evaluación del } \\
\text { curso: presentación } \\
\text { de marcomoléculas } \\
\text { seleccionadas. }\end{array}$ \\
\hline $9: 00-10: 00$ & $\begin{array}{l}\text { Presentación de } \\
\text { Curso y mecanismo } \\
\text { de evaluación. }\end{array}$ & $\begin{array}{l}\text { IV. Software PyMOL } \\
\text { y RasMOL. }\end{array}$ & $\begin{array}{l}\text { VII. Proteínas de } \\
\text { importancia en la } \\
\text { salud: } \\
\text { Hemoglobina. }\end{array}$ & $\begin{array}{l}\text { Visualización digital: } \\
\text { Enzima de } \\
\text { restricción EcoRV. }\end{array}$ & $\begin{array}{l}\text { Evaluación del } \\
\text { curso: presentación } \\
\text { de marcomoléculas } \\
\text { seleccionadas. }\end{array}$ \\
\hline 10:00-10:30 & $\begin{array}{l}\text { I. La biología y las } \\
\text { biomoléculas. }\end{array}$ & $\begin{array}{c}\text { Visualización digital } \\
\text { de } \\
\text { macromoléculas: } \\
\text { citocromo C. }\end{array}$ & $\begin{array}{c}\text { Visualización digital } \\
\text { de } \\
\text { macromoléculas: } \\
\text { oxi- y desoxi- } \\
\text { hemoglobina. }\end{array}$ & $\begin{array}{l}\text { Sesión de } \\
\text { investigación para } \\
\text { evaluación. }\end{array}$ & $\begin{array}{l}\text { Evaluación del } \\
\text { curso: presentación } \\
\text { de marcomoléculas } \\
\text { seleccionadas. }\end{array}$ \\
\hline $10: 30-11: 10$ & Receso & Receso & Receso & Receso & Receso \\
\hline 11:10-12:00 & $\begin{array}{l}\text { Instalación de } \\
\text { Software para } \\
\text { visualización de } \\
\text { biomoléculas }\end{array}$ & $\begin{array}{l}\text { V. Importancia } \\
\text { biológica de los } \\
\text { ácidos nucleicos. }\end{array}$ & $\begin{array}{l}\text { VIII. Una nueva } \\
\text { visión de las } \\
\text { enzimas como } \\
\text { nanomáquinas. }\end{array}$ & $\begin{array}{l}\text { Sesión de } \\
\text { investigación para } \\
\text { evaluación. }\end{array}$ & $\begin{array}{l}\text { Evaluación del } \\
\text { curso por los } \\
\text { asistentes y } \\
\text { conclusiones. }\end{array}$ \\
\hline 12:00-13:00 & $\begin{array}{l}\text { II. Diseño digital de } \\
\text { pequeños } \\
\text { metabolitos: } \\
\text { MARVIN y RasMol }\end{array}$ & $\begin{array}{l}\text { Visualización digital: } \\
\text { ADN y ARN. }\end{array}$ & $\begin{array}{c}\text { Visualización digital } \\
\text { de } \\
\text { macromoléculas: } \\
\text { ATP sintasa. }\end{array}$ & $\begin{array}{l}\text { Sesión de } \\
\text { investigación para } \\
\text { evaluación. }\end{array}$ & $\begin{array}{c}\text { Entrega de } \\
\text { calificaciones y } \\
\text { reconocimientos. }\end{array}$ \\
\hline 13:00-14:00 & $\begin{array}{l}\text { Ejercicios de diseño } \\
\text { de metabolitos. } \\
\text { (carbohidratos, } \\
\text { nucleótidos y } \\
\text { aminoácidos). }\end{array}$ & $\begin{array}{l}\text { VI. Proteínas de } \\
\text { importancia en la } \\
\text { salud: } \\
\text { Multicelularidad } \\
\text { insulina y } \\
\text { anticuerpos. }\end{array}$ & 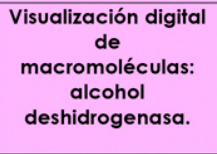 & $\begin{array}{l}\text { Sesión de } \\
\text { investigación para } \\
\text { evaluación. }\end{array}$ & Clausura de Curso \\
\hline
\end{tabular}

Figura 2. Propuesta de estrategia de trabajo de $30 \mathrm{~h}$ para el curso con tipo de sesión por color. Amarillo= administrativa formal, azul= teórica impartida por el instructor, rosa= práctica de investigación dirigida por el instructor, naranja= investigación autodirigida por los asistentes, verde $=$ presentación de conocimientos adquiridos y productos finales. Elaboración propia. 


\section{IIVESTIGAGIÓn Y GIERGIA DE LA UNIVERSIDAD AUTÓNOMA

Tabla 1

Estructuras moleculares tridimensionales autogeneradas y descargadas que se incluyen en el manual del curso y conceptos en los que pueden emplearse

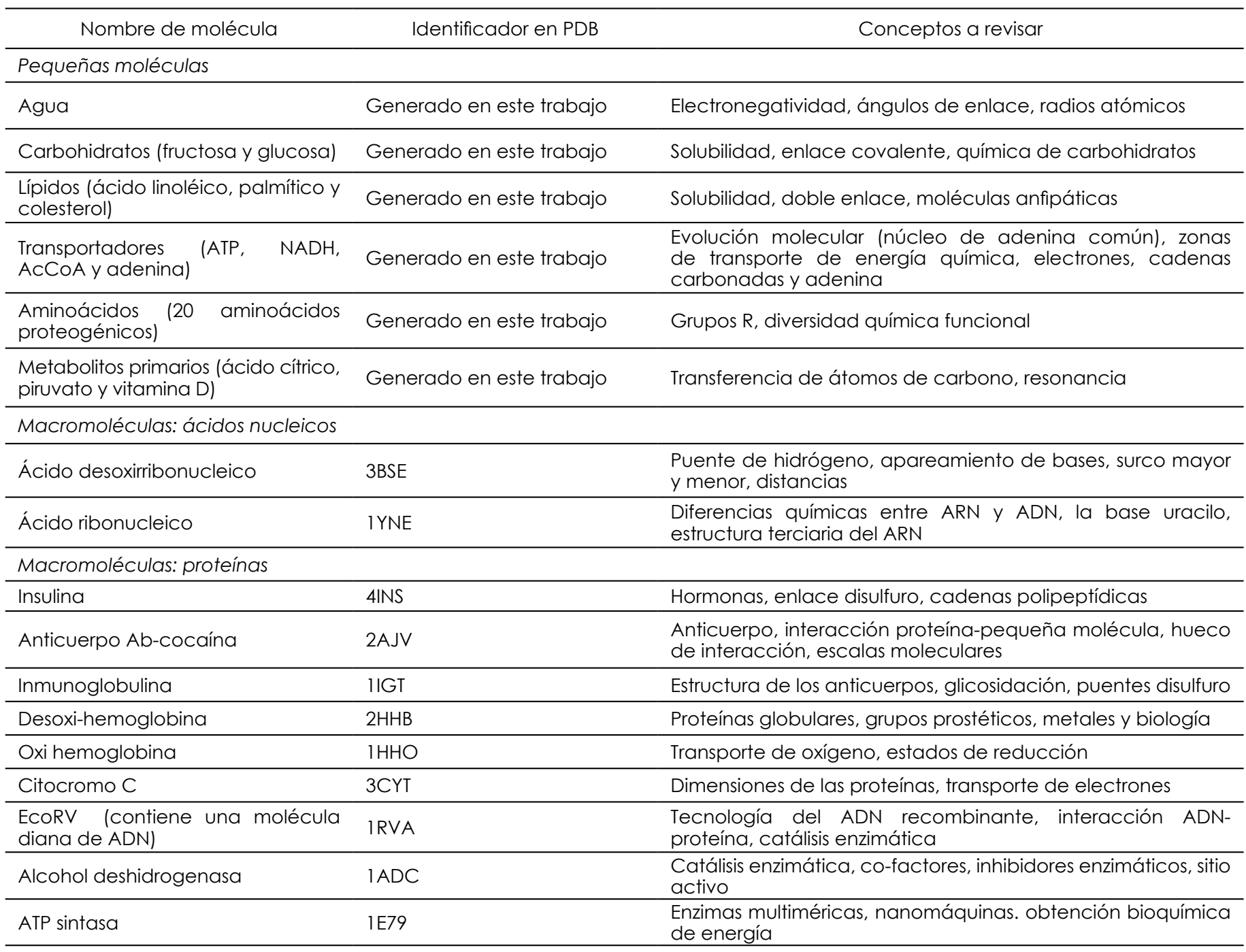

Nota: Elaboración propia.

Finalmente, el curso se evaluó con la presentación frente al grupo de un metabolito generado por el propio asistente y la visualización de las características investigadas de una proteína de su interés. Al final del curso, se aplicó una encuesta anónima en línea (Survey Monkey, 2017) a cada equipo de profesores para saber su conocimiento previo del tema, opinión sobre el potencial futuro del uso de los conocimientos adquiridos y la recepción de sugerencias.
Las moléculas que se seleccionaron para el manual y curso se dividieron en tres grupos (tabla 1): el primero fue agua y metabolitos pequeños (carbohidratos, lípidos, aminoácidos y nucleótidos), el segundo ácidos nucleicos y el tercero proteínas. El grupo de ácidos nucleicos se compuso de cadenas cortas de ácido desoxirribonucleico (ADN) y ribonucleico (ARN); el ADN también se estudia como sustrato de modificación enzimática en la sección de enzimas de restricción. El grupo de proteínas se seleccionó para reflejar sus diversos papeles biológicos como hormonas, anticuerpos, transportadores y enzimas. 
Una vez que se revisó el procedimiento para descargar e instalar los paquetes de cómputo y sus licencias académicas se hizo una revisión por el instructor del curso del papel biológico del agua y de las moléculas pequeñas con énfasis en los conceptos detallados en la tabla 1.

Se expuso Marvin Sketch y la generación de las moléculas tridimensionales (tabla 1). La sección de macromoléculas se abordó alternando pequeñas presentaciones con diapositivas que contienen los temas listados en la tabla 1 con los 12 ejercicios paso a paso del manual. Las instrucciones paso a paso fueron de fácil seguimiento por los docentes y les permitieron observar inmediatamente el éxito de sus acciones al comparar sus resultados con los resueltos en el manual. La única sesión que no incluyó práctica del concurrente fue la del uso de I-TASSER por la limitante de que sólo se puede someter una petición a la vez; el tratamiento de esta sesión se detalla en el manual.

Para la sección práctica de moléculas pequeñas los maestros se enfocaron en explorar técnicas que les ayudaran a explicar conceptos generales y estructurales básicos de química orgánica. Para las macromoléculas se abocaron a buscar proteínas de importancia en la nutrición y salud. Con ayuda del manual los presentes lograron producir imágenes diseñadas por ellos en donde se favorecieron la selección de superposiciones de modelos, el uso de la opción de transparencia y el modo cartoon (figura 3). Con estos esquemas, se enfocaron en los cuatro niveles de organización estructural de proteínas. Las selecciones de los maestros durante la sesión de investigación dirigida indicaron las necesidades que ellos tienen en el aula, pues reflejaron los temas que imparten en sus programas y pueden ayudar a diseñar nuevos manuales enfocados en estas áreas de interés.

En total se emplearon 8.5 h en sesiones teóricas (42.5\%), 8 h (40\%) en dirigidas de investigación asistida sobre moléculas descritas en los programas del IEBO y apoyadas por el manual y $3.5 \mathrm{~h}$ (17.5\%) de investigación de interés del profesor y acompañadas por el instructor a través de las bases de datos bibliográficas en línea (figura 2).

Se realizó una evaluación anónima del curso por parte de los 14 equipos de maestros que indicó que sólo en un equipo había un profesor con conocimiento previo de software para el uso en el aula (ChemDraw, Perkin Elmer, software comercial). Es decir, $96 \%$ de los asistentes nunca había estado en contacto con estas herramientas. De la encuesta también se concluyó que el manual y su implementación tuvieron objetivos claros (excelente, 100\%), que la organización temática fue adecuada (excelente, 100\%), y que los maestros consideraron que el contenido fue útil para su actualización (excelente 93\%, suficiente 7\%).

Algunas opiniones de los asistentes fueron:

Opinión 1: "Que exista en el futuro seguimiento de este curso puesto que es de gran importancia para nuestro desarrollo en la educación media superior y que obtengamos más herramientas de trabajo para el desarrollo de nuestra docencia para el educando."

Opinión 2: "Los conocimientos que se nos brindaron nos serán de gran utilidad en nuestra práctica docente."

Opinión 3: "El curso fue excelente ya que fue muy dinámico y novedoso, gracias doctor y ojalá nos dieran otro curso ya que sería en beneficio de los estudiantes."

\section{DISCUSIÓN}

Entre las opciones disponibles para diseñar cursos de visualización molecular (Vázquez-Contreras, 2015) se seleccionaron Marvin y PyMOL. Aunque son paquetes de uso profesional en publicaciones científicas, poseen una interfaz de usuario amigable. Ambos funcionaron adecuadamente incluso en equipos de bajo poder de cómputo, por lo que se espera que no sea una limitante en la sala de cómputo escolar.

En el sitio de internet donde se encuentra el manual (UNPA, s. f.) están disponibles presentaciones muestra. También como referencia para el instructor, estos conceptos se encuentran detallados en el libro en línea gratuito Structure and mechanism in protein science (Fersht, 1999). El manual del curso es esencial pues contiene guías paso a paso de uso de menús, imágenes de resultados esperados para Marvin y PyMOL, explicaciones biológicas de lo que se visualiza y referencias a los trabajos originales.

Una de las principales fortalezas educativas de los paquetes informáticos de simulación es la de sustituir en el alumno el papel de espectador por uno de participante (Badotti et al., 2014; Vázquez- 


\section{INVESTIGAGIÓก Y CIECEIA DE LA UחIVERSIDAD AUTÓกOMA DE RGUASCALIERTES}
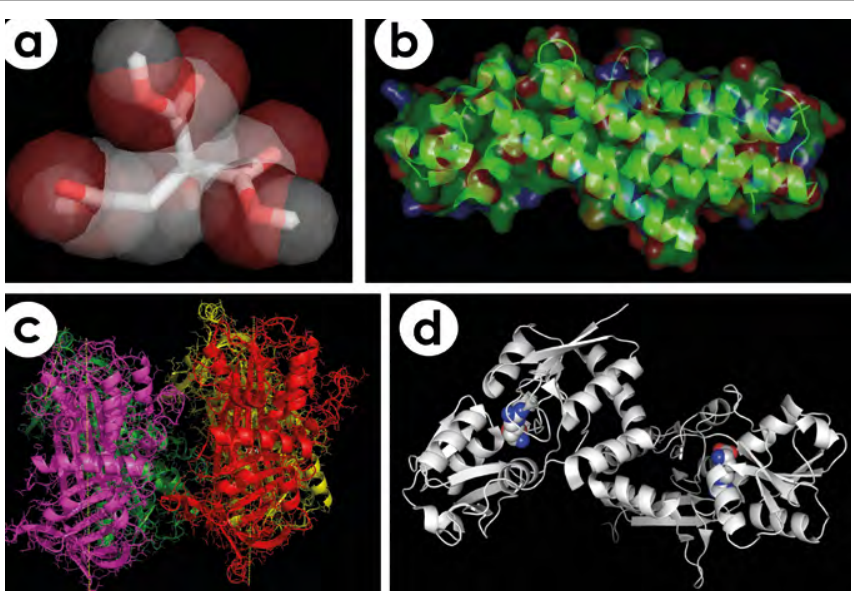

Figura 3. Ejemplos de estructuras generadas en PyMOL como producto final de evaluación. (a) Reconstrucción digital del ácido cítrico, (b) identificación de estructuras secundarias en un factor de transcripción (3G1L) de Mycobacterium tuberculosis, (c) identificación de subunidades en la ovoalbúmina de pollo (1OVA), (d) visualización de sitios activos de una proteína de unión a arginina (2Y7I) de M. tuberculosis.

Elaboración propia.

Contreras, 2015; White et al., 2010). Para ello se dedicó $57 \%$ del tiempo total a sesiones de práctica e investigación en bases de datos en línea de temas de interés de los docentes. Se explicó el concepto de artículo científico, base bibliográfica y la búsqueda de los números ID en el PDB. Se hizo especial énfasis en el uso del portal educativo del RCSB PDB (s. f.) como una fuente para encontrar temas actuales $y$ archivos PDB asociados.

El curso culminó con una evaluación de dos actividades: a) la exposición de la importancia biológica o aplicativa de una molécula pequeña y su modelo tridimensional original generado con Marvin, y b) la investigación de una macromolécula de interés e imágenes originales producidas mediante el uso de PyMOL. Como se observa en la figura 3, los productos desarrollados por los profesores del curso muestran una diversidad de aproximaciones al uso de la visualización molecular. Esto es más relevante si se toma en cuenta que $98 \%$ de los concurrentes nunca había empleado estas herramientas.

Este dato confirmó que existe una brecha entre el uso extenso de estas técnicas de diseño y visualización en ambientes científicos profesionales de tiempo completo y el conocimiento de su existencia y aplicabilidad en los maestros de nivel bachillerato. La calidad de los productos de investigación de los presentes demostró que su preparación profesional en química y biología, en conjunto con el uso del manual y la estrategia de implementación, resultaron óptimas para habilitarlos en el uso de las herramientas presentadas en este estudio.

\section{CONCLUSIONES}

El manual de los mecanismos seleccionados y su estrategia de implementación en forma de curso (figura 4) se desarrollaron para hacer accesibles de manera guiada a los asistentes dos paquetes de diseño y visualización molecular (Marvin y PyMOL), bases de datos (PDB, NCBI, Web of Science, Scopus) y un servidor (I-TASSER). Con la implementación del curso pudimos observar que la selección de dichas herramientas permitió al asistente desarrollar imágenes didácticas originales de su interés y útiles para su práctica docente.

\section{Agradecimientos}

Los autores desean agradecer a Schrödinger LLC y a ChemAxon por su generosa asignación de licencias académicas. A la Vice-Rectoría de la UNPA y al Departamento de Docencia del IEBO por la oportunidad de impartir el curso. A la Lic. Dolores Rivera Nieto y al Ing. Luis Fernando López Castillo por su asistencia técnica.

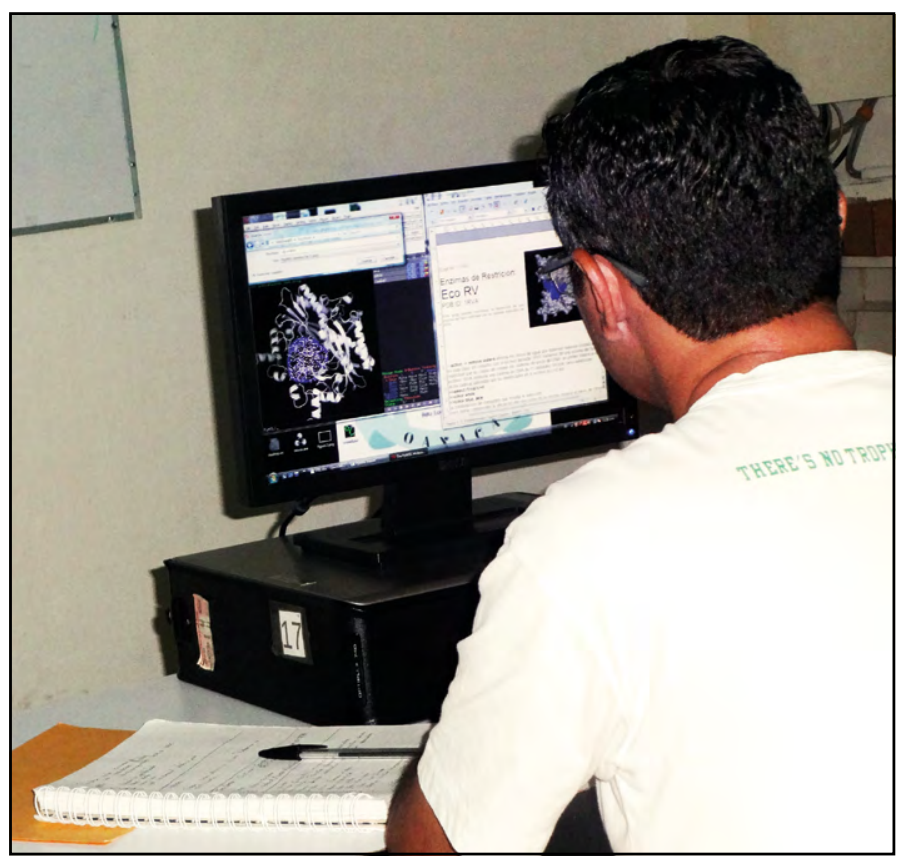

Figura 4. Profesor de nivel bachillerato utilizando herramientas de visualización molecular para realizar ejercicios de modelos didácticos digitales de proteínas.

Fotografía proporcionada por los autores. 
- Badotti, F., Barbosa, A. S., Reis, A. L. M., do Valle, I. F., Ambrósio, L., \& Bitar, M. (2014). Comparative modeling of proteins: A method for engaging students' interest in bioinformatics tools. Biochemistry and Molecular Biology Education, 42(1), 68-78. doi: 10.1002/bmb.20721

- Berman, H. M., Westbrook, J., Feng, Z., Gilliland, G., Bhat, T. N., Weissig, H., ... Bourne, P. E. (2000). The Protein Data Bank. Nucleic Acids Research, 28(1), 235-242. doi: 10.1093/ nar/28.1.235

- ChemAxon LTD. (s. f.). Download Marvin Suite (software). Recuperado de http://www.chemaxon.com/download/ Marvin-suite/\#mbeans

- Delano, W. L., \& Bromberg, S. (2004). PyMOL user's guide. Recuperado de pymol.sourceforge.net/newman/userman. pdf

- Fersht, A. (1999). Structure and mechanism in protein science: a guide to enzyme catalysis and protein folding. ( $2^{\text {nd }}$ ed.). New York, US: W. H. Freeman and Company. Recuperado de http://www.fersht.com/Structure.html

- Gonçalves de Almeida, V. M. (2012). Ferramentas para visualização de biomoléculas (PyMoL). Recuperado de www. Ibs.dcc.ufmg.br/cvbioinfo/material/PYMOL.pdf

- Hernández-Santos, W. J., Mendoza, N., Barrera-Figueroa, B. E., \& Peña-Castro, J.M. (2016). Visualización de biomoléculas con herramientas computacionales [Manual]. Oaxaca, México: Universidad del Papaloapan. Recuperado de http://www. unpa.edu.mx/ julianpc/manual_molecular/manual_PyMol_ Marvin_ISBN.pdf

- Marvin (Versión 16.3.21) [Software de computación]. Budapest, Hungary: ChemAxon.

- National Center for Biotechnology Information. (s. f.). [Base de datos]. Recuperado de www.ncbi.nlm.nih.gov

- Peña-Castro, J. M., Gregorio-Ramírez, O., \& Barrera-Figueroa, B. E. (2013). Los métodos experimentales que permiten el estudio de las macromoléculas de la vida: historia, fundamentos y perspectivas. Educación Química, 24 (2), 237-246. doi: 10.1016/ s0187-893X(13)72468-6

- RCSB PDB-101 (Educational portal of PDB). (s. f.). Molecular explorations through biology and medicine [Portal]. Recuperado de https://pdb101.rcsb.org/
- Reyes-Trejo, L. J. (2013). Taller Introducción al modelado molecular. México: UNAM. Recuperado de http://depa. fquim.unam.mx/amyd/archivero/Taller_de_Spartan_24109. pdf

- Sánchez-Murcia, P. A., \& Gago-Badenas, F. (2016). Breve introducción al programa de gráficos moleculares PyMOL. España: Universidad de Alcalá. Recuperado de http://www3. uah.es/farmamol/Public/PDF_files/ManualPyMOL.pdf

- Schrödinger LLC. (s. f.). Registration for educational-useonly PyMOL builds [Formulario de registro]. Schrödinger LLC. Recuperado de http://PyMOL.org/edu

- Scopus (s. f.). Document search [Base de datos]. Recuperado de https://www.scopus.com/home.uri

- Survey Monkey (2017). [Portal de encuestas en línea]. Recuperado de https://es.surveymonkey.com/

- Universidad del Papaloapan. (s. f.). Index of / julianpc/ manual_molecular [Base de datos]. Recuperado de http:// www.unpa.edu.mx/ julianpc/manual_molecular/

- Vázquez-Contreras, E. (2015). El uso de visualizadores moleculares en la enseñanza de la bioquímica en la UAM Cuajimalpa. En C. R. Jaimez-González, K. S. Miranda-Campos, M. Moranchel-Pocaterra, E. Vázquez-Contreras, \& F. VázquezVela (Eds.), Innovación educativa y apropiación tecnológica: experiencias docentes con el uso de las TIC (pp. 193-212). México: Universidad Autónoma Metropolitana.

- Web of Science (2017). [Base de datos]. Recuperado de webofknowledge.com

- White, B., Kahriman, A., Luberice, L., \& Idleh, F. (2010). Evaluation of software for introducing protein structure: Visualization and simulation. Biochemistry and Molecular Biology Education, 38(5), 284-289. doi: 10.1002/bmb.20410

- Worldwide Protein Data Bank. (s. f.). [Portal electrónico]. Recuperado de http://www.pdb.org

- Yang, J., Yan, R., Roy, A., Xu, D., Poisson, J., \& Zhang, Y. (2014). The I-TASSER SUite: protein structure and function prediction. Nature Methods, 12, 7-8. doi: 10.1038/nmeth.3213

- Zhang LAB (2017). I-TASSER protein structure \& function predictions [Portal de servidor]. MI, US: Zhang Lab. Recuperado de http://zhanglab.ccmb.med.umich.edu/I-TASSER/ 